\title{
Phonon spectrum and dynamical stability of a quantum degenerate Bose-Fermi mixture
}

\author{
Han $\mathrm{Pu},{ }^{1}$ Weiping Zhang, ${ }^{1}$ Martin Wilkens, ${ }^{2}$ and Pierre Meystre ${ }^{1}$ \\ ${ }^{1}$ Optical Sciences Center, The University of Arizona, Tucson, AZ 85721 \\ ${ }^{2}$ Universität Potsdam, Institut für Physik, Am Neuen Palais 10, 14469 Potsdam, Germany
}

(October 25, 2018)

\begin{abstract}
We calculate the phonon excitation spectrum in a zero-temperature boson-fermion mixture. We show how the sound velocity changes due to the boson-fermion interaction and we determine the dynamical stability regime of a homogeneous mixture. We identify a resonant phonon-exchange interaction between the fermions as the physical mechanism leading to the instability.

PACS numbers: 03.75.Fi, 05.30.Fk, 67.40.Db
\end{abstract}

New developments in the field of ultracold atoms are creating opportunities for detailed studies of quantum degenerate Fermi gases [1,2]. The Pauli exclusion principle results in the lack of $s$-wave scattering and the suppression of higher order scattering processes at low temperatures, so that the direct evaporative cooling spinpolarized fermionic atoms does not work. To circumvent this obstacle, one may either cool a Fermi gas containing two different spin states [1] or mix the fermions with a gas of bosonic atoms and sympathetically cool this mixture [2]. Both methods have been successfully implemented experimentally to create degenerate Fermi gases. The experimental progress along the latter direction has stimulated considerable renewed interest in studying BoseFermi mixtures in the quantum degenerate regime $[36$.

The study of ultracold Bose-Fermi mixtures dates back to the $1960 \mathrm{~s}$, when the discovery that ${ }^{3} \mathrm{He}$ is miscible in ${ }^{4} \mathrm{He}$ prompted the study of dilute solutions of ${ }^{3} \mathrm{He}$ in superfluid ${ }^{4} \mathrm{He}$ [7]. In that system, however, the physical picture is complicated by the strong interatomic interactions. As such, weakly-interacting alkali atomic vapor quantum gases provide us much cleaner systems to study the properties of Bose-Fermi mixtures. In this paper, we analyze the spectrum of bosonic elementary excitations in a Bose-Fermi mixture at zero temperature. In the case of an homogeneous system, we identify the stability regime of the mixture and calculate the sound velocity of the bosonic phonons under the influence of the fermions. We identify the physical mechanism leading to the instability of the mixture as a resonant phonon-exchange coupling between fermions below and above the Fermi sea.

Our starting point is the Hamiltonian describing the interaction between a bosonic and spin-polarized fermionic system

$$
\begin{aligned}
\hat{H} & =\int d^{3} r \hat{\psi}_{B}^{\dagger}\left(\hat{H}_{B}^{(0)}-\mu_{B}+\frac{g}{2} \hat{\psi}_{B}^{\dagger} \hat{\psi}_{B}\right) \hat{\psi}_{B} \\
& +\int d^{3} r \hat{\psi}_{F}^{\dagger}\left(\hat{H}_{F}^{(0)}-\mu_{F}\right) \hat{\psi}_{F}+f \int d^{3} r \hat{\psi}_{B}^{\dagger} \hat{\psi}_{B} \hat{\psi}_{F}^{\dagger} \hat{\psi}_{F}
\end{aligned}
$$

where $\hat{H}_{\alpha}^{(0)} \equiv \hat{T}_{\alpha}+V_{\alpha}(\alpha=B, F)$ and the subscripts $B$ and $F$ represent 'boson' and 'fermion', respectively; $\hat{T}_{\alpha}$, $V_{\alpha}$ and $\mu_{\alpha}$ are the associated kinetic energy, trapping potential, and chemical potential, and $g$ and $f$ represent the boson-boson and boson-fermion interaction strengths. In terms of the $s$-wave scattering lengths $a$, they are given by

$$
g=4 \pi \hbar^{2} a_{B} / m_{B}, \quad f=2 \pi \hbar^{2} a_{B F} / m_{r},
$$

with $m_{r}=m_{B} m_{F} /\left(m_{B}+m_{F}\right)$ being the reduced mass. All higher order scattering processes are neglected.

To determine the excitation spectrum of the Bose condensate, we decompose the field operators as

$$
\begin{aligned}
\hat{\psi}_{B}(\mathbf{r}, t) & =\phi_{B}(\mathbf{r})+\hat{\xi}_{B}(\mathbf{r}, t), \\
\hat{\psi}_{F}^{\dagger}(\mathbf{r}, t) \hat{\psi}_{F}(\mathbf{r}, t) & =n_{F}(\mathbf{r})+\delta \hat{\rho}_{F}(\mathbf{r}, t),
\end{aligned}
$$

where $\phi_{B}(\mathbf{r})=\left\langle\hat{\psi}_{B}(\mathbf{r}, t)\right\rangle$ is the condensate wave function and $n_{F}(\mathbf{r})=\left\langle\hat{\psi}_{F}^{\dagger}(\mathbf{r}, t) \hat{\psi}_{F}(\mathbf{r}, t)\right\rangle$ is fermion density. Clearly, $\phi_{B}$ satisfies the Gross-Pitaevskii equation

$$
\left(\hat{H}_{B}^{(0)}+g n_{B}+f n_{F}\right) \phi_{B}=0
$$

where $n_{B}=\left|\phi_{B}\right|^{2}$.

Inserting Eqs. (2) into (1), neglecting terms involving three or more fluctuation operators yields for $\hat{\xi}_{B}(\mathbf{r}, t)$ and $\hat{\psi}_{F}(\mathbf{r}, t)$ the Heisenberg equations of motion:

$$
\begin{array}{r}
i \hbar \frac{\partial \hat{\xi}_{B}}{\partial t}=\left(\hat{H}_{B}-\mu_{B}\right) \hat{\xi}_{B}+g \phi_{B}^{2} \hat{\xi}_{B}^{\dagger}+f \phi_{B} \delta \hat{\rho}_{F}, \\
i \hbar \frac{\partial \hat{\psi}_{F}}{\partial t}=\left(\hat{H}_{F}-\mu_{F}\right) \hat{\psi}_{F}+f\left(\phi_{B} \hat{\xi}_{B}^{\dagger}+\phi_{B}^{*} \hat{\xi}_{B}\right) \hat{\psi}_{F},
\end{array}
$$

with $\hat{H}_{B}=\hat{H}_{B}^{(0)}+2 g n_{B}+f n_{F}$ and $\hat{H}_{F}=\hat{H}_{F}^{(0)}+f n_{B}$.

We first find an approximate solution of (5). Expanding $\hat{\psi}_{F}(\mathbf{r}, t)$ on the basis of eigenstates $\varphi_{n}(\mathbf{r})$, with eigenenergies $E_{n}$, of the Hamiltonian $\hat{H}_{F}$ as:

$$
\hat{\psi}_{F}(\mathbf{r}, t)=\sum_{n} \hat{a}_{n}(t) \varphi_{n}(\mathbf{r}),
$$

and inserting this expansion into Eq. (5) yields the formal solution 


$$
\begin{aligned}
\hat{\psi}_{F}(\mathbf{r}, t)= & \hat{\psi}_{F}^{(0)}(\mathbf{r}, t)-i \frac{f}{\hbar} \int_{0}^{t} d t^{\prime} \int d^{3} r^{\prime} G\left(\mathbf{r}, \mathbf{r}^{\prime}, t-t^{\prime}\right) \\
& \times \Xi\left(\mathbf{r}^{\prime}, t^{\prime}\right) \hat{\psi}_{F}\left(\mathbf{r}^{\prime}, t^{\prime}\right),
\end{aligned}
$$

where

$$
\begin{aligned}
& \hat{\psi}_{F}^{(0)}(\mathbf{r}, t)=\sum_{n} \hat{a}_{n}(0) e^{-i E_{n} t / \hbar} \varphi_{n}(\mathbf{r}), \\
& G\left(\mathbf{r}, \mathbf{r}^{\prime}, t-t^{\prime}\right) \equiv \sum_{n} e^{-i E_{n}\left(t-t^{\prime}\right) / \hbar} \varphi_{n}(\mathbf{r}) \varphi_{n}^{*}\left(\mathbf{r}^{\prime}\right), \\
& \Xi\left(\mathbf{r}^{\prime}, t^{\prime}\right)=\phi_{B}\left(\mathbf{r}^{\prime}\right) \hat{\xi}_{B}^{\dagger}\left(\mathbf{r}^{\prime}, t^{\prime}\right)+\phi_{B}^{*}\left(\mathbf{r}^{\prime}\right) \hat{\xi}_{B}\left(\mathbf{r}^{\prime}, t^{\prime}\right) .
\end{aligned}
$$

In first order, we replace $\hat{\psi}_{F}\left(\mathbf{r}^{\prime}, t^{\prime}\right)$ by $\hat{\psi}_{F}^{(0)}\left(\mathbf{r}^{\prime}, t^{\prime}\right)$ on the r.h.s. of Eq. (7), so that

$$
\begin{aligned}
\hat{\psi}_{F}(\mathbf{r}, t) \approx \hat{\psi}_{F}^{(0)}(\mathbf{r}, t)-i \frac{f}{\hbar} \int_{0}^{t} d t^{\prime} \int d^{3} r^{\prime} G\left(\mathbf{r}, \mathbf{r}^{\prime}, t-t^{\prime}\right) \\
\times \Xi\left(\mathbf{r}^{\prime}, t^{\prime}\right) \hat{\psi}_{F}^{(0)}\left(\mathbf{r}^{\prime}, t^{\prime}\right) .
\end{aligned}
$$

After some algebra, this expression yields the lowest order correction to the fermionic density in the presence of the Bose condensate as:

$$
\begin{aligned}
\delta \hat{\rho}_{F} & =\hat{\psi}_{F}^{\dagger}(\mathbf{r}, t) \hat{\psi}_{F}(\mathbf{r}, t)-n_{F}(\mathbf{r}) \\
& \approx i \frac{f}{\hbar} \int_{0}^{t} d t^{\prime} \int d^{3} r^{\prime} \mathcal{J}\left(\mathbf{r}, \mathbf{r}^{\prime}, t-t^{\prime}\right) \Xi\left(\mathbf{r}^{\prime}, t^{\prime}\right),
\end{aligned}
$$

where

$$
\begin{aligned}
\mathcal{J}\left(\mathbf{r}, \mathbf{r}^{\prime}, t-t^{\prime}\right) \equiv & G_{>}^{*}\left(\mathbf{r}, \mathbf{r}^{\prime}, t-t^{\prime}\right) G_{<}\left(\mathbf{r}, \mathbf{r}^{\prime}, t-t^{\prime}\right) \\
& -G_{>}\left(\mathbf{r}, \mathbf{r}^{\prime}, t-t^{\prime}\right) G_{<}^{*}\left(\mathbf{r}, \mathbf{r}^{\prime}, t-t^{\prime}\right), \\
G_{>}\left(\mathbf{r}, \mathbf{r}^{\prime}, t-t^{\prime}\right) \equiv & \sum_{\left\{n \mid E_{n}>\mu_{F}\right\}} e^{-i E_{n}\left(t-t^{\prime}\right) / \hbar} \varphi_{n}^{*}\left(\mathbf{r}^{\prime}\right) \varphi_{n}(\mathbf{r}),
\end{aligned}
$$

and $G_{<}$is the same as $G_{>}$, but for $E_{n} \leq \mu_{F}$. In deriving Eq. (9), we have neglected second or higher order terms in $\hat{\xi}_{B}$ and $\hat{\xi}_{B}^{\dagger}$, and we have replaced terms of the form $\hat{\psi}_{F}^{(0) \dagger} \hat{\psi}_{F}^{(0)}$ by their expectation values with respect to the ground state. For example:

$$
\hat{\psi}_{F}^{(0) \dagger}(\mathbf{r}, t) \hat{\psi}_{F}^{(0)}(\mathbf{r}, t) \approx\left\langle\hat{\psi}_{F}^{(0) \dagger}(\mathbf{r}, t) \hat{\psi}_{F}^{(0)}(\mathbf{r}, t)\right\rangle=n_{F}(\mathbf{r}) .
$$

Inserting Eq. (9) back into Eq. (4) yields the equation of motion for the boson fluctuation operator:

$$
\begin{aligned}
i \hbar \frac{\partial \hat{\xi}_{B}}{\partial t} & =\left(\hat{H}_{B}^{(0)}+2 g n_{B}+f n_{F}\right) \hat{\xi}_{B}+g \phi_{B}^{2} \hat{\xi}_{B}^{\dagger} \\
& +i \frac{f^{2}}{\hbar} \int_{0}^{t} d t^{\prime} \int d^{3} r^{\prime} \mathcal{J}\left(\mathbf{r}, \mathbf{r}^{\prime}, t-t^{\prime}\right) \Xi\left(\mathbf{r}^{\prime}, t^{\prime}\right) \phi_{B}(\mathbf{r}) .
\end{aligned}
$$

This is one of the central results of this paper. It shows that the twofold effects of fermions on the excitation of the condensate: First, the fermions change the effective mean-field potential of the boson - the $f n_{F}$ term; second, the boson fluctuations affect the fermions, which then results in a back-action onto the bosons - the last term at the r.h.s. of (10).

Consider for concreteness a homogeneous mixture of $N_{B}$ bosons and $N_{F}$ fermions confined in a box of volume $V$. In the ground state, we have $\mu_{B}=g n_{B}+f n_{F}$ and $\mu_{F}=\hbar^{2} k_{F}^{2} /\left(2 m_{F}\right)+f n_{B}$, where we have introduced the densities $n_{B}=N_{B} / V$ and $n_{F}=N_{F} / V=k_{F}^{3} /\left(6 \pi^{2}\right)$ and $k_{F}$ is the Fermi wave number. For periodic boundary conditions, the eigenstates of $\hat{H}_{F}$ are plane waves $\varphi_{\mathbf{k}}(\mathbf{r})=e^{i \mathbf{k} \cdot \mathbf{r}} / \sqrt{V}$, with eigenenergies $E_{k}=\hbar^{2} k^{2} / 2 m_{F}+$ $f n_{B}$ and $k=|\mathbf{k}|$, so that the function $\mathcal{J}\left(\mathbf{r}, \mathbf{r}^{\prime}, t-t^{\prime}\right)$ of Eq. (10) can now be explicitly expressed as:

$$
\begin{array}{r}
\mathcal{J}\left(\mathbf{r}, \mathbf{r}^{\prime}, t-t^{\prime}\right)=\frac{1}{V^{2}} \sum_{k_{n}>k_{F}} \sum_{k_{m} \leq k_{F}}\left[e^{i \frac{\hbar}{2 m_{F}}\left(k_{n}^{2}-k_{m}^{2}\right)\left(t-t^{\prime}\right)}\right. \\
\left.e^{i\left(\mathbf{k}_{\mathbf{m}}-\mathbf{k}_{\mathbf{n}}\right) \cdot \mathbf{r}} e^{-i\left(\mathbf{k}_{\mathbf{m}}-\mathbf{k}_{\mathbf{n}}\right) \cdot \mathbf{r}^{\prime}}-c . c .\right] .
\end{array}
$$

Expanding likewise $\hat{\xi}_{B}(\mathbf{r}, t)$ as:

$$
\hat{\xi}_{B}(\mathbf{r}, t)=\frac{1}{\sqrt{V}} \sum_{\mathbf{k}} \eta_{\mathbf{k}}(t) e^{i \mathbf{k} \cdot \mathbf{r}},
$$

yields from Eq. (10)

$$
i \hbar \frac{\partial \eta_{\mathbf{k}}}{\partial t}=\mathcal{L}_{B}(k) \eta_{\mathbf{k}}+g n_{B} \eta_{-\mathbf{k}}^{\dagger}+(i / \hbar) f^{2} n_{B} I(\mathbf{k}),
$$

where $\mathcal{L}_{B}(k)=\hbar^{2} k^{2} / 2 m_{B}+g n_{B}$

$$
\begin{aligned}
I(\mathbf{k})= & \frac{1}{V} \int_{0}^{t} d t^{\prime} \int d^{3} r^{\prime} \int d^{3} r e^{-i \mathbf{k} \cdot \mathbf{r}} \mathcal{J}\left(\mathbf{r}, \mathbf{r}^{\prime}, t-t^{\prime}\right) \\
& \times \sum_{\mathbf{k}^{\prime}} e^{i \mathbf{k}^{\prime} \cdot \mathbf{r}^{\prime}}\left[\eta_{-\mathbf{k}^{\prime}}^{\dagger}\left(t^{\prime}\right)+\eta_{\mathbf{k}^{\prime}}\left(t^{\prime}\right)\right] \\
= & \frac{1}{V} \int_{0}^{t} d t^{\prime} \sum_{k_{m} \leq k_{F}}\left[e^{i \frac{\hbar}{2 m_{F}}\left(\left|\mathbf{k}_{\mathbf{m}}+\mathbf{k}\right|^{2}-k_{m}^{2}\right)\left(t-t^{\prime}\right)}-c . c .\right] \\
& \times \Theta\left(\left|\mathbf{k}_{\mathbf{m}}+\mathbf{k}\right|-k_{F}\right)\left[\eta_{-\mathbf{k}}^{\dagger}\left(t^{\prime}\right)+\eta_{\mathbf{k}}\left(t^{\prime}\right)\right],
\end{aligned}
$$

and $\Theta(x)$ is the unit step function. Similarly,

$$
i \hbar \frac{\partial \eta_{-\mathbf{k}}^{\dagger}}{\partial t}=-\mathcal{L}_{B}(k) \eta_{-\mathbf{k}}^{\dagger}-g n_{B} \eta_{\mathbf{k}}-(i / \hbar) f^{2} n_{B} I(\mathbf{k}) .
$$

The two coupled integro-differential equations (11) and (12) can be solved using Laplace transformation. Denoting $\alpha_{\mathbf{k}}(s)=L\left[\eta_{\mathbf{k}}(t)\right]=\int_{0}^{\infty} d t e^{-s t} \eta_{\mathbf{k}}(t)$ and $\beta_{\mathbf{k}}(s)=$ $L\left[\eta_{-\mathbf{k}}^{\dagger}(t)\right]$, Eqs. (11) and (12) yield

$$
\begin{aligned}
i \hbar s \alpha_{\mathbf{k}}(s)= & \mathcal{L}_{B}(k) \alpha_{\mathbf{k}}(s)+g n_{B} \beta_{\mathbf{k}}(s) \\
& +i \frac{f^{2}}{\hbar} n_{B} \ell_{s}(k)\left[\alpha_{\mathbf{k}}(s)+\beta_{\mathbf{k}}(s)\right], \\
i \hbar s \beta_{\mathbf{k}}(s)=- & \mathcal{L}_{B}(k) \beta_{\mathbf{k}}(s)-g n_{B} \alpha_{\mathbf{k}}(s) \\
& -i \frac{f^{2}}{\hbar} n_{B} \ell_{s}(k)\left[\alpha_{\mathbf{k}}(s)+\beta_{\mathbf{k}}(s)\right],
\end{aligned}
$$

where 


$$
\ell_{s}(k)=\frac{1}{V} L\left[\sum_{k_{m} \leq k_{F}}^{\left|\mathbf{k}_{\mathbf{m}}+\mathbf{k}\right|>k_{F}} e^{i \Delta_{\mathbf{k}+\mathbf{k}_{\mathbf{m}}, \mathbf{k}_{\mathbf{m}}} t}-c . c .\right],
$$

where $\hbar \Delta_{\mathbf{k}+\mathbf{k}_{\mathbf{m}}, \mathbf{k}_{\mathbf{m}}}=\epsilon_{\mathbf{k}_{\mathbf{m}}+\mathbf{k}}-\epsilon_{\mathbf{k}_{\mathbf{m}}}$ with $\epsilon_{\mathbf{k}}=\hbar^{2} k^{2} / 2 m_{F}$. The excitation frequency $\omega$ is simply obtained by replacing $s$ by $i \omega+0^{+}$in Eqs. (13). Since these equations are homogeneous equations in $\alpha_{\mathbf{k}}(s)$ and $\beta_{\mathbf{k}}(s), \omega$ is determined by requiring the determinant of their coefficients to be equal to zero. These steps result in the phonon dispersion relation:

$$
\hbar^{2} \omega^{2}=\frac{\hbar^{2} k^{2}}{2 m_{B}}\left[\frac{\hbar^{2} k^{2}}{2 m_{B}}+2 g n_{B}+\frac{f^{2} n_{B}}{(2 \pi)^{3} \hbar} \ell_{\omega}(k)\right],
$$

where

$$
\begin{aligned}
\ell_{\omega}(k)= & \int d \mathbf{k}_{\mathbf{m}}\left(\frac{\Theta\left(\left|\mathbf{k}_{\mathbf{m}}+\mathbf{k}\right|-k_{F}\right)}{\omega-\Delta_{\mathbf{k}+\mathbf{k}_{\mathbf{m}}, \mathbf{k}_{\mathbf{m}}}-i 0^{+}}\right. \\
& \left.-\frac{\Theta\left(\left|\mathbf{k}_{\mathbf{m}}+\mathbf{k}\right|-k_{F}\right)}{\omega+\Delta_{\mathbf{k}+\mathbf{k}_{\mathbf{m}}, \mathbf{k}_{\mathbf{m}}}-i 0^{+}}\right) \Theta\left(k_{F}-k_{m}\right) .
\end{aligned}
$$

Here we have replaced the sum over $\mathbf{k}_{\mathbf{m}}$ by an integral. As expected the Bogoliubov spectrum of a pure condensate is recovered for $f=0$.

Equation (15) has the same form as Eq. (12.30) in Ref. [8]. In general, $\ell_{\omega}(k)$ - and hence $\omega$-may take complex values, indicating that the homogeneous state of the Bose-Fermi mixture becomes unstable. Its real part is given by the principal value of the integral in (15) and can be expressed as:

$$
\begin{aligned}
& \operatorname{Re}\left[\ell_{\omega}(k)\right]=-\frac{4 \pi m_{F} k_{F}}{\hbar}-\frac{2 \pi}{\alpha^{3}}\left[\left(\omega-\frac{\alpha k}{2}\right)^{2}-\alpha^{2} k_{F}^{2}\right] \\
& \quad \times \ln \left|\frac{\omega+\alpha\left(k_{F}-k / 2\right)}{\omega-\alpha\left(k_{F}+k / 2\right)}\right|-\frac{2 \pi}{\alpha^{3}}\left[\left(\omega+\frac{\alpha k}{2}\right)^{2}-\alpha^{2} k_{F}^{2}\right] \\
& \quad \times \ln \left|\frac{\omega-\alpha\left(k_{F}-k / 2\right)}{\omega+\alpha\left(k_{F}+k / 2\right)}\right|,
\end{aligned}
$$

with $\alpha=\hbar k / m_{F}$. Its imaginary part, which determines the stability of the mixture, is given by

$$
\begin{aligned}
\operatorname{Im}\left[\ell_{\omega}(k)\right]= & \pi \int d \mathbf{k}_{\mathbf{m}} \Theta\left(\left|\mathbf{k}_{\mathbf{m}}+\mathbf{k}\right|-k_{F}\right) \Theta\left(k_{F}-k_{m}\right) \\
& \times \delta\left(\omega-\Delta_{\mathbf{k}+\mathbf{k}_{\mathbf{m}}, \mathbf{k}_{\mathbf{m}}}\right),
\end{aligned}
$$

which is nothing but the dynamical structure factor of the Fermi gas, $S(k, \omega)$, apart from a constant factor [9].

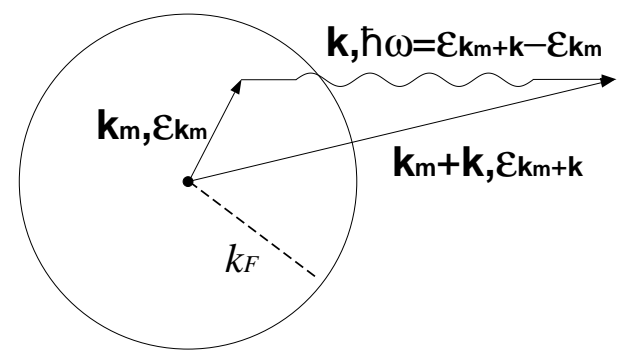

FIG. 1. Resonant phonon-exchange interactions between fermions results in dynamical instability of the system.

The physical interpretation of the instability associated with $\operatorname{Im}\left[\ell_{\omega}(k)\right] \neq 0$ [or $\left.S(k, \omega) \neq 0\right]$ is directly apparent from Eq. (16). The condition that a phonon of wave vector $\mathbf{k}$ and frequency $\omega$ satisfies $\omega=\Delta_{\mathbf{k}+\mathbf{k}_{\mathrm{m}}, \mathbf{k}_{\mathrm{m}}}$ for some $k_{m}<k_{F}$ and $\left|\mathbf{k}_{\mathbf{m}}+\mathbf{k}\right|>k_{F}$ implies that it resonantly couples a pair of fermions lying inside and above the Fermi sea, respectively, see Fig. 1. When this coupling occurs, the Bose-Fermi mixture becomes dynamically unstable, and the system will spontaneously break into several distinct phases [6]. For long-wavelength phonons $\left(k \ll k_{F}\right)$, the stability criterion determined by $\operatorname{Im}\left[\ell_{\omega}(k)\right]=0$ requires the phonon frequency to satisfy:

$$
\omega>\frac{\hbar k^{2}}{2 m_{F}}+\frac{\hbar k k_{F}}{m_{F}} .
$$

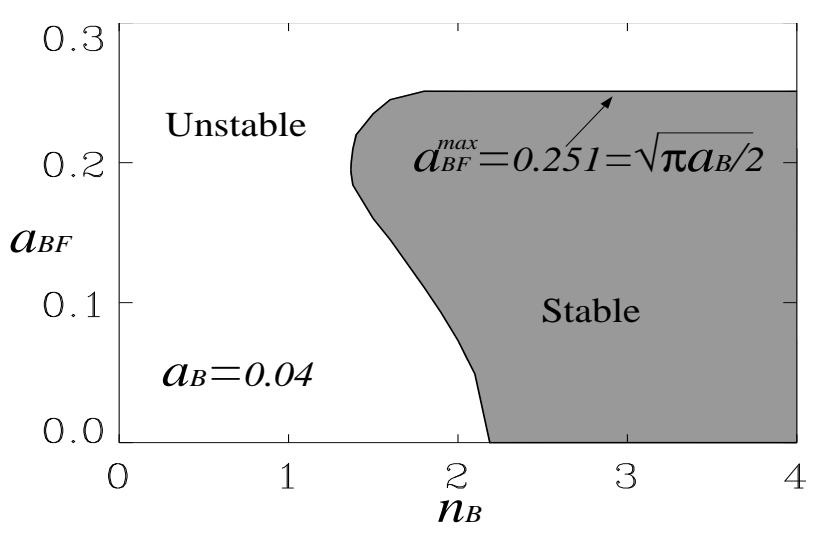

FIG. 2. Stability diagram of a Bose-Fermi mixture. We assume $m_{F}=m_{B}=m$. We have adopted a system units in which the units for frequency, length, and wavenumber are $\hbar k_{F}^{2} / 2 m, 1 / k_{F}$, and $k_{F}$, respectively. Note that in this units, the fermion density is given by $n_{F}=1 /\left(6 \pi^{2}\right) \approx 0.017$.

The stability regime and the phonon spectrum can be obtained by first solving Eq. (14) numerically while assuming $\operatorname{Im}\left[\ell_{\omega}(k)\right]=0$, and then checking if the system is stable using the criterion (17). Fig. 22 shows the stability diagram of the mixture in the $a_{B F}-n_{B}$ space. As can be seen, the dynamical stability of the system is determined by both the scattering lengths and the densities. All other parameters being fixed, the stability condition (17) imposes a minimum boson density $n_{B}^{\min }$ beyond which no stable homogeneous mixture exists. We can use the Bogoliubov spectrum of a pure condensate to estimate $n_{B}^{\min }$ as

$$
g n_{B}^{\min } \approx \frac{\hbar^{2} k_{F}^{2}}{m_{F}^{2} / m_{B}}=\frac{\hbar^{2}\left(6 \pi^{2} n_{F}\right)^{2 / 3}}{m_{F}^{2} / m_{B}} .
$$

For realistic numbers, $n_{B}^{\min }$ is about two orders of magnitude larger than $n_{F}$ (see Fig. 2). 


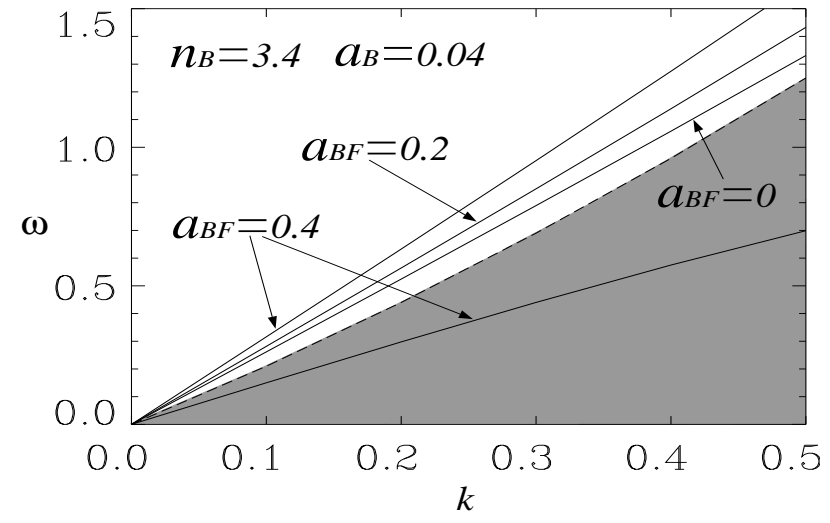

FIG. 3. Phonon spectrum of a boson-fermion mixture. The dashed line corresponds to $\omega=\hbar\left(k^{2}+2 k k_{F}\right) / 2 m_{F}$. The gray area below the dashed line represents the unstable regime. Same units as in Fig. 2.

Figure 3 illustrates the phonon spectrum in the BoseFermi mixture for $n_{B}>n_{B}^{\min }$. For small values of $a_{B F}$, the spectrum is stable and single valued. Furthermore, the boson-fermion interaction increases the sound velocity of the phonon. By expanding $\ell_{\omega}(k)$ around small $k$, it is not difficult to show that in our dimensionless units (see caption of Fig. 2) and for $m_{B}=m_{F}$ the sound velocity is approximately given by

$$
c \approx c_{0}\left[1+\frac{4 a_{B F}^{2}}{\left(2+c_{0}\right)^{2} \pi a_{B}}\right]
$$

where $c_{0}=\sqrt{16 \pi a_{B} n_{B}}$ is the sound velocity in a pure condensate. For the parameters in Fig. 3, we have a $6 \%$ increase in sound velocity if $a_{B F}$ changes from 0 to 0.2 . However, further increasing $a_{B F}$ beyond a critical value $a_{B F}^{\max }$ splits the phonon spectrum into two branches, and one of them falls into the unstable regime. Again using the expansion of $\ell_{\omega}(k)$, one can show that $a_{B F}^{\max }$ is determined by (in dimensionless units):

$$
\left(a_{B F}^{\max }\right)^{2}=2 \pi a_{B} m_{r} /\left(m_{B}+m_{F}\right) .
$$

This condition agrees with that obtained by Viverit et al. [6] and is confirmed by our numerical calculations (see Fig. 3). For $m_{F} \approx m_{B}$, we have

$$
a_{B F}^{\max }=\sqrt{\pi a_{B} / 2} .
$$

Note that while $n_{B}^{\min }$ is independent of $a_{B F}, a_{B F}^{\max }$ is independent of $n_{B}$. Putting (19) into (18), we obtain the maximum sound velocity achievable in a homogeneous mixture as:

$$
c_{\max }=c_{0}\left[1+\frac{2}{\left(2+c_{0}\right)^{2}}\right] .
$$

In conclusion, we have developed a general formalism to determine the phonon spectrum of the condensate in a Bose-Fermi mixture at zero temperature. We have shown that a resonant phonon-exchange interaction between fermions can induce a dynamical instability in the mixture. As an example, we have calculated the phonon spectrum of a homogeneous system and obtained analytically the sound velocity under the effect of boson-fermion coupling. From the spectrum, we are able to determine the dynamical stability regime of such a system which depends on both the scattering lengths and the densities, and is directly linked to the dynamical structure factor of the Fermi gas. Our calculations show that for a given fermion density, a much denser boson field is required to stabilize the mixture. Viverit et al. also considered the stability of a homogeneous mixture [6]. In contrast to our work, which depicts a full dynamic picture for the boson-fermion mixture, their interest was focused on the mechanical stability phase diagram of the system, which is obtained by requiring the total energy to be a minimum but does not take the phonon-exchange interaction into full account.

Our method can be straightforwardly applied to an inhomogeneous system, although the algebra in this case is much more involved. We will address this situation in a future publication. Another interesting case is when a boson field is mixed with a fermion field containing two components (e.g., two spin states). Interactions between the bosons and fermions will induce an effective coupling between the fermions from different components, which may result in the creation of Cooper pairs.

This work is supported in part by the US Office of Naval Research under Contract No. 14-91-J1205, by the National Science Foundation under Grant No. PHY9801099, by the US Army Research Office, and by the Joint Services Opitcs Program.One of us (MW) acknowledges support by the DFG national programme SPP 1116.

[1] B. DeMarco and D. S. Jin, Science 285, 1703 (1999).

[2] A. G. Truscott et al., Science Express Reports, March 1, 2001.

[3] K. Mølmer, Phys. Rev. Lett. 80, 1804 (1998).

[4] M. Amoruso et al., Eur. Phys. J. D 4, 261 (1998).

[5] M. J. Bijlsma, B. A. Heringa and H. T. C. Stoof, Phys. Rev. A 61, 053601 (2000).

[6] L. Viverit et al., Phys. Rev. A 61, 053605 (2000).

[7] G. Baym and C. Pethick, Landau Fermi-Liquid Theory (John Wiley \& Sons, New York, 1991).

[8] A. L. Fetter and J. D. Walecka, Quantum Theory of ManyParticle Systems (McGraw Hill, New York 1971).

[9] D. Pines and P. Nozières, The Theory of Quantum Liquids, Vol. I, (W. A. Benjamin, New York, 1966). 